\title{
Síndrome del intestino irritable: una revisión narrativa
}

\author{
Irritable bowel syndrome: a narrative review \\ Síndrome do intestino irritável: uma revisão narrativa
}

\author{
Yulissa Verdugo ${ }^{1 *}$, Adriana Márquez1. \\ Recibido: 3 de junio de 2021. Aceptado para publicación: 13 de agosto de 2021. \\ Primero en línea: 9 de septiembre 2021 \\ https://doi.org/10.35454/rncm.v4n4.306
}

\section{Resumen}

Introducción: el síndrome de intestino irritable (SII) es uno de los trastornos gastrointestinales más comunes con una prevalencia mundial que va del $10 \%$ al $15 \%$. Su tratamiento nutricional se basa en el reconocimiento de la sintomatología asociada con la ingesta de ciertos alimentos, por lo cual se han implementado diversas estrategias, entre ellas la dieta FODMAP (oligosacáridos, disacáridos, monosacáridos y polioles fermentables), mediterránea, libre de gluten e inclusión de fibra y probióticos.

Objetivo: brindar información sobre el abordaje nutricional en el SII.

Metodología: las evidencias que sustentan el presente artículo fueron obtenidas a través de búsquedas en las plataformas de Pubmed y Google Scholar con una expansión de búsqueda del año 2000 al 2021.

Resultados: diversos metaanálisis sugieren la eficacia de una dieta baja en los FODMAP como parte del tratamiento del SIl; así mismo, se sugiere que los probióticos son una terapia efectiva que contribuye a la mejoría de los síntomas generales del SIl, dolor abdominal y flatulencia.

Conclusión: tener más conocimientos sobre la etiología y fisiopatología de la enfermedad podría abrir las puertas hacia nuevos tratamientos; es imprescindible entender el papel que juega la dieta y el estilo de vida en el tratamiento del SII.

Palabras clave: síndrome del intestino irritable, colon irritable, dieta.

\section{Summary}

Introduction: Irritable bowel syndrome (IBS) is one of the most common gastrointestinal disorders, with a worldwide prevalence ranging from $10 \%$ to $15 \%$. Nutritional treatment is based on the recognition of the symptomatology associated with the intake of certain foods, hence the implementation of various strategies, including the FODMAP (fermentable oligosaccharides, disaccharides, monosaccharides and polyols), the Mediterranean, and glutenfree diets, and the inclusion of fiber and probiotics.

Objective: To provide information regarding nutritional approaches in irritable bowel syndrome.

Methodology: The evidence supporting this article was obtained through searches in the PUBMED and Google Scholar platforms, with a search expansion from 2000 to 2021.

Results: Several meta-analyses suggest the efficacy of low FODMAPs diet as part of the treatment of IBS, and also that probiotics are an effective therapy that contributes to the improvement of general IBS symptoms, abdominal pain and flatulence.

Conclusion: Improved knowledge of the etiology and pathophysiology of the disease could open the door to new treatments. It is imperative to understand the role of diet and lifestyle in the treatment of irritable bowel syndrome.

Keywords: Irritable bowel syndrome; Irritable colon; Diet.

\section{Resumo}

Introdução: A síndrome do intestino irritável é uma das doenças gastrointestinais mais comuns, com prevalência mundial que varia de $10 \%$ a $15 \%$. O seu tratamento nutricional baseia-se no reconhecimento dos sintomas associados à ingestão de determinados alimentos, para os quais várias estratégias têm sido implementadas, incluindo a dieta FODMAP, mediterrânica, sem glúten e inclusão de fibras e probióticos.

Objetivo: fornecer informações sobre a abordagem nutricional na Síndrome do intestino irritável.

Metodologia: as evidências que sustentam este artigo foram obtidas por meio de revisão bibliográfica, utilizando as plataformas PUBMED e Google acadêmico, Os artigos seleccionados foram publicados no período entre 2000 e 2021.

Resultados: várias meta-análises sugerem a eficácia de uma dieta baixa em FODMAPs como parte do tratamento do SII, da mesma forma, sugere-se que os probióticos são uma terapia eficaz que contribui para a melhora dos sintomas gerais do SII, dor abdominal e flatulência.

Conclusão: O aprimoramento dos conhecimentos sobre a etiologia e fisiopatologia da doença poderiam abrir portas para novos tratamentos, sendo fundamental o entendimento do papel que a dieta e o estilo de vida desempenham no tratamento da síndrome do intestino irritável.

Palavras-chave: síndrome do intestino irritável, colón irritável, dieta.
División de Ciencias Biológicas y de la Salud, Departamento de Ciencias de la Salud, Universidad de Sonora. Sonora, México.
*Correspondencia: Yulissa Verdugo.

yu.lagarda@gmail.com 


\section{INTRODUCCIÓN}

El síndrome de intestino irritable (SII) es uno de los trastornos gastrointestinales más comunes con una prevalencia mundial que va del $9,2 \%$ al $15 \%^{(1,2)}$. Es una patología crónica cuya sintomatología se caracteriza por ser recurrente, acompañada de dolor abdominal, distensión y cambios en la defecación que se pueden presentar con predominio de diarrea, estreñimiento o ambas, y con base en esto se clasificará como SII con predominio de diarrea (SII-D), SII con predominio de estreñimiento (SII-C) o SII mixto (SII-M), en el cual se presenta tanto diarrea como estreñimiento ${ }^{(1)}$. Estas condiciones se manifiestan aun cuando no hay otra situación patológica que desencadene los síntomas.

La etiología de la enfermedad no está del todo dilucidada, pero se sabe que múltiples comorbilidades como los síndromes de dolor somático, desórdenes gastrointestinales y psiquiátricos incrementan la posibilidad del desarrollo de la patología ${ }^{(3)}$. Así mismo, se ha evidenciado que la ingesta de alimentos genera síntomas en 9 de cada 10 pacientes con SII y que algunos pueden agravar la sintomatología. Algunos pacientes muestran mejoría de los síntomas cuando se restringen ciertos alimentos, modificando patrones de alimentación, como la incorporación de la dieta FODMAP (oligosacáridos, disacáridos, monosacáridos y polioles fermentables), libre de gluten, dieta mediterránea, inclusión de fibra o ciertas cepas de probióticos que se han asociado con la mejoría de síntomas como distención y flatulencia.

Para el abordaje nutricional en los pacientes con SII se tienen algunas opciones que son discutidas en el presente artículo; así mismo, es importante señalar que los principales retos van enfocados en la adherencia al tratamiento y las diferencias que pueden existir en la respuesta al abordaje nutricional según el subtipo de SII de cada paciente.

El objetivo de la presente revisión es dar un panorama general de la patología y el abordaje nutricional para la mejoría de los síntomas en los pacientes con SII.

\section{ANÁLISIS Y DISCUSIÓN}

\section{Metodología de búsqueda}

Las evidencias que sustentan el presente artículo fueron obtenidas a través de búsquedas en la plataforma de Pubmed y Google Scholar. Los términos de búsqueda fueron los siguientes: irritable bowel syndrome, phatophysiology irritable bowel syndrome, irritable bowel syndrome nutritional treatment, medical treatment irritable bowel syndrome, síndrome del intestino irritable, gluten free diet and irritable bowel syndrome, probiotics and irritable bowel syndrome, mediterranean diet for irritable bowel syndorme. En las bases de datos, el rango de expansión para la búsqueda de la información fue del 2000 al 2021. La mayoría de los artículos recabados se encontró en inglés, y una minoría en español. Se seleccionaron 47 artículos y se consideraron todos aquellos que estuvieron relacionados con los términos de búsqueda, el rango de expansión y cuyo contenido estuviese enfocado al objetivo del presente estudio.

\section{Definición}

El SII es una afección médica con características de dolor abdominal, distención abdominal, malestar abdominal recurrente y crónico, hábitos intestinales alterados (como el estreñimiento, diarrea o ambos), con ausencia de una enfermedad orgánica gastrointestinal asociada ${ }^{(1)}$. El inicio de la sintomatología debe ocurrir al menos 6 meses antes de efectuarse el diagnóstico y los síntomas deben estar presentes durante al menos 3 meses $^{(4)}$.

\section{Prevalencia}

La prevalencia del SII es muy variada ya que depende de los criterios diagnósticos utilizados y la metodología ${ }^{(2)}$. En general, se tiene una prevalencia mundial que va del $9,2 \%$ al $15 \%{ }^{(1)}$; sin embargo, en un metaanálisis publicado en el $2020^{(2)}$ se evidenció una prevalencia combinada del SII en 53 estudios que utilizaron los criterios de Roma III, de 9,2 \% (intervalo de confianza [IC] del $95 \%$ : 7,6-10,8; índice de inconsistencia $\left[\mathrm{I}^{2}\right]=99,7 \%$ ) y la prevalencia combinada de SII en 6 estudios que utilizaron los criterios de Roma IV de 3,8 \% (IC $95 \%$ : $\left.3,1-4,5 ; I^{2}=96,6 \%\right)^{(2)}$; así mismo, se identificó que la prevalencia varió entre países y esta variabilidad persistió incluso cuando se aplicaron los mismos criterios diagnósticos y se utilizó una metodología idéntica en los estudios ${ }^{(2)}$.

De todas las consultas al área de gastroenterología, del $25 \%$ al $50 \%$ corresponden al SII ${ }^{(1)}$. En México, el SII afecta del $10 \%$ al $20 \%$ de la población ${ }^{(5)}$ y, debido a que su prevalencia es muy elevada, tiene afectaciones socioeconómicas graves ${ }^{(5)}$. Este trastorno gastrointestinal se puede presentar en cualquier etapa de la vida; sin embargo, las probabilidades aumentan en el grupo de edad de los 30 a 50 años ${ }^{(6)}$. Afecta mayormente a mujeres que a hombres con una prevalencia de $2: 1^{(4)}$. La prevalencia de las diferentes presentaciones de SII 
es muy variable, ya que el SII-C se presenta en 5,2 \% a $66 \%$ de los pacientes, el SII-D en $0,8 \%$ al 33,9 \% y el SII-M en $5,2 \%$ a $33,8 \%{ }^{(2,6)}$.

\section{Fisiopatología}

La etiología y fisiopatología del SII no está del todo dilucidada; sin embargo, se sabe que algunos factores pueden incrementar el riesgo de desarrollar esta condición (Tabla 1$)^{(7)}$. Tradicionalmente, el SII se considera un síndrome biopsicosocial. Se ha sugerido que los cambios que se dan entre la comunicación cerebrointestino son secundarios a alteraciones psicológicas, las cuales condicionan a una anormalidad en la motilidad e hipersensibilidad intestinal ${ }^{(8)}$. No obstante, no todas las personas que desarrollan este síndrome presentan condiciones psicológicas importantes que los lleve al desarrollo de la sintomatología ${ }^{(9,10)}$. Se han establecido algunas hipótesis de explicaciones fisiopatológicas propuestas sobre la causa del SII que han permitido el desarrollo de algunas técnicas terapéuticas, dentro de las cuales se encuentran la disregulación en los niveles de serotonina, disregulación en la motilidad intestinal, inflamación, posinfecciones, disfunciones psicosociales y factores genéticos ${ }^{(1)}$.

Tabla 1. Factores etiológicos asociados con SII

\begin{tabular}{|l|l|}
\hline \multicolumn{1}{|c|}{ Etiología } & \multicolumn{1}{c|}{ Características } \\
\hline Demográficos & $\begin{array}{l}\text { Sexo femenino } \\
>50 \text { años }\end{array}$ \\
\hline Factores psicológicos & $\begin{array}{l}\text { Baja calidad de vida } \\
\text { Estrés } \\
\text { Historia de abuso sexual } \\
\text { Ansiedad } \\
\text { Depresión }\end{array}$ \\
\hline Enfermedades somáticas & $\begin{array}{l}\text { Infección gastrointestinal } \\
\text { Obesidad abdominal } \\
\text { Endometriosis } \\
\text { Cirugía de abdomen } \\
\text { Uso de antibióticos }\end{array}$ \\
\hline
\end{tabular}

Adaptada y modificada de ${ }^{(7)}$.

\section{Infección y activación inmune en el SII}

Se ha demostrado que los pacientes con SII tienen niveles de mastocitos y linfocitos más altos que los pacientes sanos, así como también niveles de citocinas alterados y una permeabilidad intestinal anormal ${ }^{(11)}$. Estudios prospectivos han encontrado que del $3 \%$ al $36 \%$ de las enfermedades bacterianas y parasitarias trasmisi- bles conducen a síntomas propios del SII ${ }^{(12)}$. También se ha asociado con el proceso posinfeccioso como un desencadenante del síndrome y dentro de los factores de riesgo para el desarrollo de SII posinfeccioso se encuentran la duración de la enfermedad inicial, la toxicidad de la bacteria causante de la infección, consumo de tabaco, sexo femenino y presentar algún desorden psicológico como depresión o ansiedad. No se conoce la causa exacta por la cual un proceso posinfeccioso causa el SII, pero se piensa que se debe a residuos de la inflamación ${ }^{(13)}$.

\section{Disregulación en los niveles de serotonina}

Se ha observado que la concentración de serotonina en plasma está aumentada en personas con SII-D y disminuida en aquellos con SII-C ${ }^{(14)}$. Esto es de especial interés ya que, a través de receptores, principalmente los receptores 3 y 4 de 5-hidroxitriptamina (5-HT3 y 5-HT4), la serotonina participa de manera muy importante en la función gastrointestinal influyendo tanto en la sensación visceral como en la motilidad y secreción intestinal.

\section{Intolerancia alimentaria}

La mayoría de los pacientes identifica ciertos alimentos como los desencadenantes de la sintomatología y las intolerancias alimentarias son muy comunes ${ }^{(3)}$. Los pacientes con SII refieren no tolerar de manera adecuada la lactosa, ya que lo asocian con el inicio de diarrea y distensión abdominal. Además, se ha visto que las personas con SII tienen un mayor riesgo de padecer intolerancia a la lactosa que la población sana ${ }^{(15)}$.

En cuanto a los alimentos que se relacionan con el inicio de la sintomatología, estos se han denominado como los FODMAP, acrónimo que hace referencia a carbohidratos altamente fermentables como los oligosacáridos, disacáridos, monosacáridos y polioles. Los alimentos con alto contenido en FODMAP tienen 3 características principales que podrían ser la causa de la sintomatología: son muy pobremente absorbidos por el intestino delgado, son moléculas osmóticamente activas que atraen líquidos de manera rápida hacia la luz intestinal y son altamente fermentables por bacterias del colon, lo que produce una gran cantidad de gases. Como resultado final de la intolerancia a los alimentos ricos en FODMAP se tiene la distensión abdominal, dolor abdominal, diarrea y exceso de gases ${ }^{(6)}$. Los factores psicosociales como el estrés, ansiedad y depresión han mostrado ser un importante predisponente del SII ya que ocasionan una disfunción en la comunicación 
cerebro-intestino, lo que puede ser permanente y traducirse en trastornos gastrointestinales ${ }^{(3)}$. Los factores genéticos también parecen jugar un rol importante en el desarrollo de la enfermedad (Tabla 1$)^{(9)}$.

\section{Factores genéticos}

Estudios en gemelos en distintas regiones del mundo han mostrado mayores tasas de concordancia entre monocigóticos en comparación con gemelos dicigóticos, lo que probablemente sugiere un componente genético en el SII ${ }^{(12,16)}$. Se ha observado una influencia positiva a la presencia de SII cuando se tienen familiares directos con el síndrome. Han sido varios los genes candidatos en el estudio de SII. La molécula más estudiada es la serotonina (5-hidroxitriptamina [5-HT]) por el papel que tiene en el eje cerebrointestino y su concentración en el tracto gastrointestinal. Existe evidencia de que los pacientes con SII tienen defectos en la señalización serotoninérgica; en consecuencia, se han investigado polimorfismos en el gen del transportador de recaptación de serotonina (SERT) $)^{(12,16)}$. En el artículo "Genetic epidemiology of irritable bowel syndrome", Makker hace referencia al estudio de Lesch, en el que se señala que el genotipo $\mathrm{S} / \mathrm{S}$, en comparación con los genotipos L/L y S/L, tenía una menor captación de 5-HT, lo que resultó en niveles sanguíneos más altos de $5-\mathrm{HT}^{(12,16)}$.

Otros genes que han sido estudiados en su relación con SII son aquellos afines con citocinas inflamatorias como el factor de necrosis tumoral alfa (TNF- $\alpha$ ), factor de crecimiento transformante beta 1 (TGF- $\left.\beta_{1}\right)$, interleucina 8 (IL-8) e interleucina 10 (IL-10); de los polimorfismos estudiados, para el gen de la IL-10 en la posición 1082, se observa que el alelo $\mathrm{G}$ se asocia con una alta producción de IL-10, mientras que el alelo A se asocia con una baja producción de la misma. Para el TNF- $\alpha$, alelo A y TGF- $\beta_{1}$, los alelos T y G se asocian con una alta producción de estas citocinas proinflamatorias aumentadas en $\mathrm{SII}^{(12,16)}$.

El $\Delta$-9-tetrahidrocannabinol, ingrediente activo del cannabis, actúa por medio de receptores canabinoides (CB1 y $\mathrm{CB} 2$ ), los receptores $\mathrm{CB} 1$ tienen presencia en todo el tracto gastrointestinal y el genotipo AAT, con más de 10 repeticiones del gen del receptor CB1, que se encontró asociado con la presencia de SII. También se ha estudiado el polimorfismo (rs324420) del gen de la enzima amida hidrolasa del ácido graso (FAAH) y se observó que el genotipo A/A se encontraba con menos frecuencia en sujetos con SII frente a los controles sanos; sin embargo, las diferencias no fueron significativas ${ }^{(12,16)}$.
Se han estudiado algunos polimorfismos de nucleótido único (SNP) de diversos genes como el factor de necrosis tumoral super familia 15 (TNFSF15), colecistoquinina 1 (CCK-1), catecol-O-metiltransferasa (COMT), proteína fijadora de nucleótido de guanina (GN $\beta 3)$, entre otros, los mismos que sugieren la asociación genética en SII, pero es importante reconocer que aún se continúan explorando genes candidatos y se piensa que los factores ambientales probablemente tengan un mayor papel en la patogenia de la enfermedad ${ }^{(12,16)}$.

\section{Diagnóstico}

El diagnóstico debe basarse en 4 características: historia clínica, examen físico, pruebas de laboratorio mínimas y, cuando sea indicado, colonoscopia o pruebas adicionales $^{(4)}$.

Para el diagnóstico se considera la presencia de los síntomas típicos de este síndrome ${ }^{(3)}$. Actualmente, el estándar de oro para el diagnóstico de SII es los criterios de Roma IV, los cuales se basan en la presencia de dolor abdominal y cambio en las deposiciones (Tabla $2)^{(4)}$. Estos criterios permiten caracterizar a los diferentes subtipos de SII (Tabla 3) ${ }^{(4)}$. El esfuerzo excesivo al defecar, la presencia de moco en las heces y la urgencia por defecar se presentan con frecuencia en el SII ${ }^{(1)}$. Además, el $80 \%$ de los pacientes presenta distención abdominal y sensación de plenitud ${ }^{(1)}$. Algunos pacientes con SII pueden presentar diarrea, estreñimiento y en ocasiones una combinación de ambas. Es importante identificar de manera correcta el subtipo de SII del que se trata, ya que de eso depende la selección del tratamiento médico.

\section{Tabla 2. Criterios de diagnóstico Roma IV para SII}

Dolor abdominal recurrente, en promedio al menos 1 día por semana en los últimos 3 meses, asociado con 2 o más de los siguientes criterios:

alteraciones de la defecación

cambio en la frecuencia de las deposiciones

cambio en la forma (apariencia) de heces

Criterios cumplidos durante los últimos 3 meses.

Inicio al menos 6 meses antes del diagnóstico.

Adaptada y modificada de ${ }^{(4)}$.

\section{Tratamiento médico}

El tratamiento debe iniciar explicando al paciente la condición, la historia natural benigna, educación sobre 
Tabla 3. Clasificación del SIl de acuerdo con el patrón predominante de evacuaciones

\begin{tabular}{|c|c|}
\hline Subtipos de SII & Criterios de diagnóstico \\
\hline $\begin{array}{l}\text { SIl con estreñimiento } \\
\text { predominante (SII-C) }\end{array}$ & $\begin{array}{l}\text { Heces duras }>25 \% \text { de las veces (escala de Bristol tipos } 1 \text { y } 2 \text { ) y acuosas en menos del } 25 \% \text { de } \\
\text { las evacuaciones (escala de Bristol } 6 \text { y } 7 \text { ) }\end{array}$ \\
\hline $\begin{array}{l}\text { SIl con diarrea predominante } \\
\text { (SII-D) }\end{array}$ & $\begin{array}{l}\text { Heces sueltas o acuosas }>25 \% \text { (escala de Bristol } 6 \text { y } 7 \text { ) y duras en menos del } 25 \% \text { de las } \\
\text { evacuaciones (escala de Bristol tipos } 1 \text { y } 2 \text { ) }\end{array}$ \\
\hline SII mixto (SII-M) & $\begin{array}{l}\text { Heces duras }>25 \% \text { (escala de Bristol tipo } 1 \text { y } 2 \text { ) y acuosas }>25 \% \text { de las evacuaciones (escala } \\
\text { de Bristol } 6 \text { y } 7 \text { ) }\end{array}$ \\
\hline SII no clasificado & $\begin{array}{l}\text { - Pacientes que cumplen con los criterios diagnósticos para SII, pero cuyos hábitos intestinales } \\
\text { no pueden ser categorizados en los subgrupos anteriores } \\
\text { - Anormalidad insuficiente en la consistencia de las evacuaciones para completar los criterios } \\
\text { de SII-C, SII-D o SII-M }\end{array}$ \\
\hline \multicolumn{2}{|c|}{$\begin{array}{l}\text { Solo se pueden establecer con seguridad cuando el paciente es evaluado sin medicamentos para tratar las anomalías en los } \\
\text { hábitos intestinales. }\end{array}$} \\
\hline
\end{tabular}

Adaptada y modificada de ${ }^{(4)}$.

la utilidad y seguridad de las pruebas diagnósticas y opciones de tratamiento ${ }^{(4)}$. El abordaje debe ser integral y enfocarse en la mejoría de la calidad de vida del paciente y el alivio de la sintomatología clásica como la diarrea o estreñimiento, distensión y dolor abdominal $^{(17)}$. Los medicamentos que se utilizan para la mejoría de los síntomas generales son:

- Antiespasmódicos: los espasmos son uno de los principales síntomas referidos por los pacientes con SII. Los antiespasmódicos son un grupo de sustancias que actúan interrumpiendo los espasmos en el músculo liso intersticial ${ }^{(18)}$. Se utilizan como tratamiento de primera línea para la mejoría de los síntomas de espasmos y dolor abdominal (Tabla 4). Comúnmente se recomienda que la toma de estos medicamentos sea posprandial.

- Antibióticos: a pesar de que la fisiopatología del SII no se conoce del todo, hay evidencia que correlaciona la sobrepoblación bacteriana con el desarrollo de la patología de al menos una parte de estos pacientes, y que este sobrecrecimiento bacteriano podría jugar un rol importante en las alteraciones de motilidad e hipersensibilidad intestinal, así como también en la activación inmunitaria de estos sujetos ${ }^{(19)}$. La rifaximina es un antibiótico oral de amplio espectro, pobremente absorbido (menor al $1 \%$ ) y tiene una actividad gastrointestinal local ${ }^{(20)}$. Ha mostrado mejorar los síntomas generales del SII en pacientes con y sin sobrecrecimiento bacteriano (Tabla 4) ${ }^{(17)}$. La seguridad y efectividad de la rifaximina ya ha sido evaluada $^{(21)}$. Este no se toma diariamente, sino que la forma más común de administración es la toma de $550 \mathrm{mg} 3$ veces al día por 14 días. Sin embargo, su eficacia podría verse disminuida con el tiempo y será necesario repetir la dosis.

- Antidepresivos: se indican en pacientes que muestran refractariedad con antiespasmódicos y cambios en la dieta (Tabla 4). Se necesita de algunas semanas para que surjan efecto y la dosis utilizada es mucho menor a la que se indica para el tratamiento de la depresión ${ }^{(22)}$.

\section{Tratamiento del SII-C}

Los pacientes con SII con predominio de estreñimiento frecuentemente requieren la administración de laxantes para la mejoría de este problema. Existen distintos tipos de laxantes y estos se clasifican de acuerdo con su mecanismo de acción y propiedades químicas ${ }^{(23)}$. Los laxantes osmóticos como el polietilenglicol pueden ayudar a que los pacientes con SII-C aumenten la frecuencia de los movimientos intestinales $\mathrm{y}$, de este modo, mejoren el estreñimiento ${ }^{(22)}$, aunque no parece contribuir con la mejoría del dolor y distensión abdominal ${ }^{(24)}$. En adultos mayores y en pacientes con enfermedad renal, los laxantes osmóticos hipertónicos deben utilizarse con precaución ya que pueden ocasionar un desequilibro electrolítico ${ }^{(22)}$. La lactulosa es un laxante osmótico que debe evitarse en pacientes con SII ya que produce exceso de gases y puede exacerbar la sintomatología de la inflamación. En la Tabla 5 se muestra un resumen de los principales medicamentos utilizados en el tratamiento del SII-C. 
Tabla 4. Tratamiento médico para los síntomas generales del SII

\begin{tabular}{|l|l|l|l|}
\hline \multicolumn{1}{|c|}{ Ejemplo } & \multicolumn{1}{|c|}{ Mecanismo de acción } & \multicolumn{1}{c|}{ Beneficios } & \multicolumn{1}{c|}{ Efectos secundarios } \\
\hline $\begin{array}{l}\text { Antiespasmódicos: diciclomina, } \\
\text { hiosciamina y aceite de menta }\end{array}$ & $\begin{array}{l}\text { Reduce la peristalsis e } \\
\text { hipersensibilidad. }\end{array}$ & $\begin{array}{l}\text { Alivia los espasmos y } \\
\text { calambres estomacales } \\
\text { comunes en el SIl. }\end{array}$ & Boca seca, mareos, visión borrosa \\
\hline Antidepresivos: tricíclicos, ISRS & $\begin{array}{l}\text { Además de mejorar el } \\
\text { estado de ánimo, puede } \\
\text { tener efectos analgésicos. }\end{array}$ & $\begin{array}{l}\text { Mejora de manera global } \\
\text { la sintomatología. }\end{array}$ & $\begin{array}{l}\text { - Sequedad en la boca, somnolencia } \\
\text { - Los tricíclicos pueden propiciar } \\
\text { el estreñimiento al disminuir el } \\
\text { tránsito intestinal }\end{array}$ \\
\hline Antibióticos: rifaximina & $\begin{array}{l}\text { Genera cambios en la } \\
\text { microbiota intestinal. }\end{array}$ & $\begin{array}{l}\text { Mejora de manera global } \\
\text { la sintomatología. }\end{array}$ & Sin efectos secundarios \\
\hline
\end{tabular}

ISRS: inhibidores selectivos de la recaptación de serotonina. Adaptada y modificada de ${ }^{(22)}$.

Tabla 5. Tratamiento médico para los síntomas del SII-C

\begin{tabular}{|l|l|l|l|}
\hline \multicolumn{1}{|c|}{ Ejemplo } & \multicolumn{1}{|c|}{ Mecanismo de acción } & \multicolumn{1}{c|}{ Beneficios } & \multicolumn{1}{c|}{$\begin{array}{c}\text { Efectos } \\
\text { secundarios }\end{array}$} \\
\hline $\begin{array}{l}\text { Laxantes osmóticos: } \\
\text { polietilenglicol }\end{array}$ & $\begin{array}{l}\text { Provoca retención de agua en } \\
\text { el colon, lo que promueve el } \\
\text { ablandamiento de las heces y el } \\
\text { incremento en la defecación. }\end{array}$ & $\begin{array}{l}\text { Medicamento de primera línea. Efectivo para } \\
\text { la mejoría del estreñimiento, mejoría de la } \\
\text { consistencia de las heces, aumento de las } \\
\text { defecaciones y disminución del esfuerzo al defecar. }\end{array}$ & $\begin{array}{l}\text { Es bien tolerado, } \\
\text { aunque puede } \\
\text { empeorar la } \\
\text { inflamación. }\end{array}$ \\
\hline $\begin{array}{l}\text { Agonista de la } \\
\text { guanilato ciclasa C: } \\
\text { linaclotida }\end{array}$ & $\begin{array}{l}\text { Produce una aceleración del tránsito } \\
\text { intestinal al inducir la secreción de } \\
\text { cloroy bicarbonato. }\end{array}$ & $\begin{array}{l}\text { Medicamento de segunda línea. Reduce el dolor } \\
\text { abdominal, mejora la defecación y los síntomas } \\
\text { generales. }\end{array}$ & Diarrea \\
\hline
\end{tabular}

Adaptada y modificada de ${ }^{(22)}$.

\section{Tratamiento del SII-D}

Los pacientes con SII con predominio de diarrea tienen evacuaciones frecuentes cuya consistencia puede llegar a ser líquida, por lo cual el tratamiento estará enfocado en la prescripción de fármacos que reduzcan el tránsito intestinal ${ }^{(25)}$. En México, dentro de los agentes antidiarreicos más utilizados está la loperamida, que inhibe la peristalsis y disminuye el tránsito intestinal, y se considera un medicamento de primera línea para el tratamiento del SII-D (Tabla 6). Su toma se indica dependiendo de la gravedad y frecuencia de los síntomas. El alosetrón es otro medicamento útil ya que reduce el dolor, el tránsito intestinal y la diarrea. El eluxadoline fue aprobado en el 2015 como tratamiento para el SII-D ${ }^{(26)}$ y la dosis recomendada es de $100 \mathrm{mg} 2$ veces al día acompañado con comida.

\section{El papel del ejercicio en el SII}

Es bien conocido que el ejercicio disminuye los niveles de estrés, lo cual se asocia con la mejoría de los síntomas del SII ${ }^{(12)}$ dada la relación cebro-intestino; por tanto, puede ayudar a aliviar algunos síntomas asociados con SII. Se ha reportado que pedalear una bicicleta puede mejorar los síntomas y algunos estudios han reportado que alivia la distención por presencia de gases.

Además del ejercicio, la práctica de yoga ha mostrado reducir los síntomas del SII en poblaciones de distintas edades, esta actividad se ha asociado con el aumento del tono simpático, que está disminuido en IBS-D ${ }^{(27)}$.

\section{Tratamiento nutricional}

La comida genera síntomas en 9 de cada 10 pacientes con $\mathrm{SII}^{(28)}$ y ciertos alimentos parecen agravar la sintomatología ${ }^{(29)}$. Dos terceras partes de los pacientes muestran mejorar sus síntomas cuando inician una dieta restringida en alimentos y que incrementan su nivel de actividad física ${ }^{(26)}$. Existen estudios publicados sobre intervenciones dietéticas llevadas a cabo en pacientes con SII, pero estos han mostrado ser generalmente débiles y se recomienda un mayor grado de evidencia para definir a cierto tipo de dieta como la indicada para 
Tabla 6. Tratamiento médico para los síntomas del SII-D

\begin{tabular}{|l|l|l|l|}
\hline \multicolumn{1}{|c|}{ Ejemplo } & \multicolumn{1}{|c|}{ Mecanismo de acción } & \multicolumn{1}{c|}{ Beneficios } & \multicolumn{1}{c|}{ Efectos secundarios } \\
\hline Antidiarreico: loperamida & $\begin{array}{l}\text { Inhibe la peristalsis, lo que permite un } \\
\text { aumento en el tránsito intestinal y una } \\
\text { reducción en la frecuencia de defecación. }\end{array}$ & $\begin{array}{l}\text { Medicamento de primera } \\
\text { línea en SII-D y SII-M. Reduce } \\
\text { la frecuencia en la defecación. }\end{array}$ & $\begin{array}{l}\text { Generalmente bien } \\
\text { tolerado }\end{array}$ \\
\hline $\begin{array}{l}\text { Selectivo 5-HT3 antagonista } \\
\text { del receptor: alosetrón }\end{array}$ & $\begin{array}{l}\text { Disminuye la secreción y motilidad } \\
\text { colónica. }\end{array}$ & $\begin{array}{l}\text { Mejora los síntomas generales } \\
\text { y la consistencia de las heces. }\end{array}$ & $\begin{array}{l}\text { Colitis isquémica, } \\
\text { estreñimiento grave }\end{array}$ \\
\hline Eluxadoline & $\begin{array}{l}\text { Disminuye el tránsito gastrointestinal y el } \\
\text { dolor visceral. }\end{array}$ & $\begin{array}{l}\text { Mejora el dolor abdominal y } \\
\text { la diarrea. }\end{array}$ & $\begin{array}{l}\text { Náuseas, } \\
\text { estreñimiento, } \\
\text { dolor abdominal, } \\
\text { pancreatitis }\end{array}$ \\
\hline
\end{tabular}

Adaptada y modificada de ${ }^{(22)}$.

el tratamiento del SII ${ }^{(29)}$. Según las directrices de la Asociación Dietética Británica (BDA), se recomienda como primera línea del tratamiento una alimentación saludable y el control de estilo de vida ${ }^{(30)}$.

A pesar de esto, diversos metaanálisis sugieren la eficacia de una dieta baja en FODMAP como parte del tratamiento del SII ${ }^{(29,31)}$.

\section{Dieta baja en FODMAP}

Es un tipo de dieta en la cual se restringen alimentos que contienen oligosacáridos, disacáridos, monosacáridos y polioles. Estas sustancias químicas, al ser pobremente absorbidas, inducen efectos osmóticos o se fermentan en el intestino, lo cual puede exacerbar aún más la sintomatología al propiciar la distensión y secreción de flui$\operatorname{dos}^{(26)}$. Cuando se sigue una dieta baja en FODMAP, la ingesta de fibra suele ser inferior a la de una dieta habitual ${ }^{(26)}$. También existe evidencia de que este tipo de dieta reduce la ingesta de calcio a corto plazo $^{(26)}$. Según las directrices de la BDA, se recomienda la dieta FODPMAP como una segunda línea en el tratamiento nutricional del paciente con $\mathrm{SII}^{(32)}$.

La fase de restricción de una dieta baja en FODMAP que se sugiere al principio es de 3 a 6 semanas $^{(26)}$. Después de este tiempo, si se ha conseguido una mejoría en la sintomatología, se deberá continuar con la reintroducción de alimentos con contenido de FODMAP y esto se hará de manera progresiva dependiendo de la tolerancia del paciente y utilizando la estrategia que el nutricionista indique. Una técnica que puede resultar eficaz es reintroducir primero los alimentos con bajo contenido en FODMAP; después, aquellos con mediano contenido e ir reintroduciendo a los de mayor contenido de manera paulatina. Es fundamental realizar esta transición en la reintroducción de alimentos al paciente, porque favorece el apego al tratamiento, brinda una mayor diversidad dietética y, por ende, más acceso dietético a micronutrientes que pueden verse mermados en dietas muy restrictivas.

El nutriólogo debe otorgar información oral y escrita al paciente para que este comprenda la importancia del autocuidado en el SII, así como también brindar información sobre su nuevo estilo de vida, actividad física y alimentación. Además, se le debe enseñar al paciente a identificar entre los alimentos con alto y bajo contenido en FODMAP(26).

La implementación de una dieta baja en FODMAP se realiza en tres fases o etapas: restricción, reintroducción y personalización.

\section{Fase de restricción}

Esta fase consiste en disminuir de manera drástica el consumo de los FODMAP. El médico y dietista deben hacer énfasis en explicar al paciente que no se trata de una dieta de por vida, sino que es solamente una etapa del tratamiento y que el objetivo que se busca al evitar todos los FODMAP es disminuir al mínimo la sintomatología ${ }^{(33)}$. Se debe capacitar al paciente para que logre identificar los alimentos FODMAP, así como también para que logre identificar si se trata de un alimento con bajo, mediano o alto contenido en estos. El paciente debe comprender que la restricción de los FODMAP será su estilo de vida dietético durante esta fase, que incluye desde la planificación de compras hasta la realización de algunas actividades sociales y que el éxito del tratamiento está determinado por la adherencia que él logre ${ }^{(34)}$. Se 
sugiere que la duración de esta fase sea de 4 semanas $^{(35)}$, porque se ha visto que la mayoría de los pacientes logra mejorar los síntomas en este tiempo; sin embargo, la duración podría variar de un paciente a otro y se puede llegar a extender si los síntomas no mejoran ${ }^{(36)}$.

\section{Fase de reintroducción}

La reintroducción de los FODMAP se realiza de forma paulatina y con dosis controladas que dependen de la tolerancia del individuo ${ }^{(34)}$. La meta que se pretende alcanzar en esta fase es que el paciente logre identificar aquellos FODMAP que puede tolerar y aquellos que no, o que puede tolerar solo en pequeñas dosis. Se considera una fase muy importante porque el paciente no debería seguir una dieta tan restringida por mucho tiempo debido a que se ha mostrado que afecta la microbiota intestinal y el estado nutricional del paciente ${ }^{(37,38)}$. La implementación de esta fase generalmente se realiza de la semana 4 a la 12; es decir, el inicio será después de terminar la cuarta semana de la fase de restricción ${ }^{(34)}$.

En la cita de seguimiento se debe evaluar la composición corporal del individuo debido a que algunos estudios han mostrado que los pacientes pierden peso durante la fase de restricción ${ }^{(34)}$. Debe realizarse un interrogatorio al paciente con la finalidad de evaluar los cambios en la sintomatología que se han obtenido después de la restricción de los FODMAP, ya que de esto dependerá la dirección que tome el tratamiento dietético. Si los síntomas han mejorado después de la fase de restricción, se puede proseguir con esta segunda fase; pero si no es así, se tendrá que continuar con la fase de restricción hasta que se muestre una mejoría ${ }^{(34)}$. $\mathrm{Al}$ igual que en la fase anterior, en esta el paciente también debe ser instruido sobre la reintroducción de los FODMAP. Para iniciar esta fase no existe un protocolo específico, pues no hay suficientes investigaciones que indiquen el orden ideal que se debería seguir para la reintroducción de alimentos y esto podría ser debido a que la tolerancia de los distintos FODMAP varía de un paciente a otro; sin embargo, se sugiere que se consideren las preferencias dietéticas del individuo ${ }^{(39)}$. De forma general, se incluye al pan, ajo y cebolla para probar con el grupo de los fructanos, al albaricoque para probar con el sorbitol, al mango como parte de fructosa, lentejas en galactooligosacáridos, leche para probar con lactosa, hongos para el manitol y alimentos que contienen una combinación de FODMAP ${ }^{(39)}$.

Una forma práctica de experimentar con los distintos FODMAP es tomar de cada grupo al alimento con mayor contenido; por ejemplo, al probar con la fructosa se elige al mango y, si el paciente lo tolera, se considera que se podrá tolerar a todos los alimentos de ese mismo grupo. Aunque este método podría ser aplicable para los diferentes grupos de FODMAP, no es así para los fructanos, puesto que los distintos alimentos que se encuentran en este grupo difieren en su grado de fermentación debido a su estructura molecular, por lo cual no se podría tomar a un alimento como referencia sino a varios ${ }^{(40)}$.

La reintroducción de los FODMAP se lleva a cabo de forma paulatina. El proceso consiste en que el paciente sea sometido a un alimento con alto contenido en un FODMAP durante 3 días en dosis crecientes y, en este tiempo, se deben restringir a los demás alimentos FODMAP. Si durante el primer día de prueba los síntomas se exacerbaron y en los siguientes días se agravan aún más, el paciente puede decidir evitar ese grupo de alimentos por completo o consumirlos en pequeñas dosis de manera esporádica. Si el alimento no provoca síntomas molestos, este se puede incluir sin problema dentro de la dieta del paciente y pasar a la siguiente prueba con un grupo distinto ${ }^{(34)}$. Es importante que siempre se considere la calidad de vida que está teniendo el paciente $y$, si la exclusión de cierto grupo de alimentos la afecta de manera grave, se debe conseguir llegar a un acuerdo para que el paciente disminuya su estrés y eso contribuya con la mejoría de los síntomas.

\section{Fase de personalización}

La meta de esta etapa es lograr que el paciente pueda ampliar la variedad de su dieta y a la vez mejorar sus síntomas, lo cual contribuirá a mejorar su calidad de vida. Aunque la dieta será restringida en algunos FODMAP, en esta fase ya se incluirán a todos aquellos alimentos que durante la fase de reintroducción no causaron síntomas ${ }^{(34)}$. La diversidad de alimentos que el paciente podrá incluir en esta fase depende de su tolerancia, pero se debe hacer un esfuerzo por lograr una dieta lo más cercana posible a lo normal, agradable para el paciente y que cumpla con los 5 criterios de una dieta adecuada: suficiente, variada, adecuada, inocua y equilibrada.

\section{Fibra}

La adición de fibra de Psillyum ha mostrado ayudar a la mejoría de los síntomas de algunos pacientes con SII$\mathrm{C}^{(41,42)}$. Se ha visto que la ingesta adicional de fibra soluble (psyllium/cáscara de ispágula) mejora de manera moderada la sintomatología de pacientes con SII con predominio de estreñimiento, por lo cual podría ser empleada como parte del tratamiento ${ }^{(4)}$. Por su parte, 
el consumo de fibra insoluble no ha mostrado tener un efecto significativo en la mejoría global de los síntomas.

\section{Dieta libre de gluten y dieta mediterránea}

Aunque la implementación de una dieta baja en FODMAP es el método más utilizado como parte del tratamiento del SII, se ha demostrado que hasta un 30 $\%$ de los pacientes no responde y no muestra mejoría significativa en sus síntomas ${ }^{(43)}$. Por lo anterior, se ha tenido que evaluar el efecto de otro tipo de dietas en estos pacientes. En 2020 se reportó una revisión de la literatura en la que se analizaron investigaciones que compararon la eficacia de una dieta baja en FODMAP frente a la eficacia de distintos regímenes alimenticios como la implementación de una dieta libre de gluten y una dieta mediterránea ${ }^{(43)}$. Los resultados de la intervención muestran que tanto la dieta mediterránea como la libre de gluten son tan eficaces para la mejoría de los síntomas generales del SII como lo es la dieta baja en FODMAP, pues no se mostró una diferencia significativa entre ellas ${ }^{(44)}$. Sin embargo, solo con la dieta baja en FODMAP se logró alcanzar la escala 4 de Bristol y también muestra superioridad en la mejoría de los síntomas gastrointestinales individuales.

La evidencia aún es contradictoria sobre los beneficios de una dieta libre en gluten en pacientes con $\mathrm{SII}^{(45)}$. Según las directrices de la BDA, este tipo de dieta no es indicada para esta patología ${ }^{(32)}$, aún faltan más estudios con rigor metodológico que proporcionen bases para poder recomendar esta dieta dentro del tratamiento nutricional.

En la Tabla 7 se muestran distintas opciones para el abordaje nutricional en pacientes con SII.

\section{Probióticos}

En el SII existe un fuerte desorden gastrointestinal y las evidencias sugieren que una disfunción en la microbiota intestinal podría estar fuertemente relacionado con ello. En una revisión sistemática y metaanálisis se concluyó que los probióticos son una terapia efectiva que contribuye a la mejoría de los síntomas generales del SII, dolor abdominal y flatulencia ${ }^{(44)}$. Aunque la eficacia del uso de cepas y grupos individuales aún no es clara, las evidencias que sustentaron ese estudio se basaron en el uso de combinaciones de probióticos como L. plantarum 299v (cepa DSM 9843), E. coli (DSM 1752) y E. faecium. Las combinaciones de probióticos y L. plantarum DSM 9843 obtuvieron la mayor cantidad de evidencia ${ }^{(44)}$. El Consenso mexicano sobre probióticos en gastroenterología recomienda el uso de probióticos para el abordaje global de los síntomas del SII en población adulta ${ }^{(45)}$.

\section{CONCLUSIÓN}

El SII se define como una disfunción en la comunicación cerebro-intestino que lleva a una disfunción gastrointestinal regulada por factores como el estrés, dieta y condiciones gastrointestinales. La distensión, dolor abdominal y presencia de diarrea o estreñimiento son las principales características del SII. Actualmente se utilizan los criterios de Roma IV para el diagnóstico; sin embargo, se espera que con el avance de la ciencia se puedan crear biomarcadores más específicos que permitan diferenciar de manera más clara entre el SII y la presencia de cualquier otra enfermedad orgánica ${ }^{(29)}$.

Se sugiere que la primera línea de acción en el abordaje nutricional esté enfocado a una alimentación saludable y cambios en el estilo de vida ${ }^{(32)}$. Sin embargo, algunos metaanálisis sugieren la efectividad de la dieta FODMAP como parte del tratamiento del $\mathrm{SII}^{(29)}$ y el National Institute for Health and Care Excellence of the United Kingdom apoya la idea de una dieta baja en FODMAP como primera línea de tratamiento, aunque existe controversia sobre si su uso se debe realizar en todos los pacientes debido a los posibles déficits nutricionales que conlleva y que hasta un $30 \%$ de los pacientes no responde a este tratamiento.

El uso de una combinación de probióticos como L. plantarum 299v (DSM 9843), E. coli DSM 1752 y E. faecium también ha mostrado mejorar los síntomas generales del SII, dolor abdominal y flatulencia.

Aun con todos los avances en opciones dietéticas, es necesario realizar más estudios que puedan evidenciar nuevos conocimientos sobre el beneficio de patrones de alimentación específicos y la aplicación en el área clínica.

\section{PUNTOS CLAVE}

- Criterios ROMA IV.

- Clasificación del SII de acuerdo con el patrón predominante de evacuaciones.

- Tratamiento nutricional sugerido en los pacientes con SII.

- Abordaje de una dieta baja en FODMAP.

- Implementación de las fases del tratamiento nutricional. 
Tabla 7. Opciones para el abordaje nutricional en el paciente con SII

\begin{tabular}{|c|c|}
\hline $\begin{array}{l}\text { Dieta } \\
\text { FODMAP }\end{array}$ & $\begin{array}{l}\text { Es un tipo de dieta en la cual se restringen alimentos que contienen oligosacáridos, disacáridos, monosacáridos y } \\
\text { polioles. } \\
\text { Diversos metaanálisis sugieren la eficacia de una dieta baja en FODMAP como parte del tratamiento del SII }{ }^{(28)} \text {. } \\
\text { El nutriólogo debe brindar educación nutricional y ayudar al paciente a comprender e implementar una dieta } \\
\text { baja en FODMAP. } \\
\text { Consiste en tres fases: } \\
\text { - Fase de restricción: se eliminan los FODMAP por un período de } 4 \text { a } 6 \text { semanas. } \\
\text { - Fase de reintroducción: se reintroducen en la dieta los FODMAP de manera paulatina y en dosis controladas } \\
\text { - } \text { para determinar la tolerancia del paciente y tratar de conseguir la inclusión de todos los alimentos posibles. } \\
\text { a la vez mejorar sus síntomas, lo cual contribuirá a mejorar su calidad de vida. } \\
\text { Aunque la dieta será restringida en algunos FODMAP, en esta fase ya se incluirán todos aquellos alimentos que } \\
\text { durante la fase de reintroducción no causaron síntomas. }\end{array}$ \\
\hline $\begin{array}{l}\text { Dieta } \\
\text { mediterránea }\end{array}$ & $\begin{array}{l}\text { Se considera una dieta aliada para la salud y se ha demostrado que puede contribuir con la mejoría de los } \\
\text { síntomas generales del SII }{ }^{(44)} \text {. } \\
\text { La dieta mediterránea consiste en consumir }{ }^{(46)} \text { : } \\
\text { - } 3 \text { o más porciones de verduras crudas y cocidas al día } \\
\text { - } 2 \text { o más porciones de fruta al día } \\
\text { - } \text { aceite de oliva de } 3 \text { a } 6 \text { cucharadas al día } \\
\text { - productos lácteos de } 2 \text { a } 4 \text { porciones al día } \\
\text { - cereales, principalmente pan y pasta de } 3 \text { a } 4 \text { veces al día } \\
\text { - legumbres al menos } 3 \text { veces por semana } \\
\text { - frutos secos al menos } 3 \text { veces por semana } \\
\text { - huevos de } 1 \text { a } 4 \text { porciones por semana } \\
\text { - pescados y mariscos de } 2 \text { a } 4 \text { veces por semana } \\
\text { - carnes rojas } 1 \text { vez por semana o menos } \\
\text { - } \text { aves de } 2 \text { a } 4 \text { veces por semana } \\
\text { Se ha visto que la ingesta adicional de fibra soluble (psyllium/cáscara ispágula) mejora de manera moderada la } \\
\text { sintomatología de pacientes con SIl con predominio de estreñimiento, por lo cual podría ser empleada como } \\
\text { parte del tratamiento }{ }^{(4)} \text {. }\end{array}$ \\
\hline $\begin{array}{l}\text { Dieta libre de } \\
\text { gluten }\end{array}$ & $\begin{array}{l}\text { Se ha demostrado que una dieta libre de gluten puede contribuir a la mejoría de los síntomas generales del SII(44). } \\
\text { Se le denomina "gluten" a un grupo de proteínas del trigo compuestas por gliadinas y gluteninas. } \\
\text { Una dieta libre de gluten consiste en evitar el consumo de todos los alimentos que lo contienen. } \\
\text { Algunos de los alimentos que se deben evitar(47): } \\
\text { - } \text { trigo, cebada, centeno, espelta, kamut, triticale, harinas, pan, pasta y galletas con gluten; } \\
\text { - verduras y hortalizas congeladas, precocinadas, cremas de verduras industriales; } \\
\text { - legumbres en conserva o granel, papas prefritas congeladas; } \\
\text { - } \text { - fruta deshidratada, mermeladas, frutos secos; } \\
\text { - carnes preparadas con salsa o elaboración no controlada, hamburguesas, embutidos; } \\
\text { - pescados y mariscos preparados o precocinados; } \\
\text { - margarinas, leche o yogur en polvo, quesos manipulados; } \\
\text { - salsas y sopas comerciales; } \\
\text { - mezclas de especias o especias a granel; } \\
\text { - levaduras químicas; } \\
\text { - chocolate; } \\
\text { - licores cremosos, cervezas sin especificar; } \\
\text { - café soluble o en cápsulas; } \\
\text { - gelatinas de colores. } \\
\text { En una revisión sistemática y metaanálisis se concluyó que los probióticos son una terapia efectiva que contribuye } \\
\text { con la mejoría de los síntomas generales del SII, dolor abdominal y flatulencia }{ }^{(44)} \text {. Aunque la eficacia del uso } \\
\text { de cepas y grupos individuales aún no es clara, las evidencias que sustentaron ese estudio se basaron en el } \\
\text { uso de combinaciones de probióticos como Lactobacillus plantarum DSM } 9843 \text {, Escherichia coli DSM } 1752 \text { y } \\
\text { Enterococcus faecium. Las combinaciones de probióticos y L. plantarum DSM } 9843 \text { obtuvieron la mayor cantidad } \\
\text { de evidencia(44). }\end{array}$ \\
\hline
\end{tabular}




\section{Relevancia clínica}

El presente artículo define la importancia de la actualización en la práctica clínica sobre SII, porque, aunque se desconoce completamente la etiología y fisiopatología, es relevante diferenciar entre los tipos de SII para un mejor abordaje médico y nutricional.

\section{Agradecimientos}

Ninguno.

\section{Declaración de autoría}

Y. Verdugo y A. Márquez contribuyeron igualmente en la concepción de la investigación; Y. Verdugo y A. Márquez contribuyeron igualmente en la redacción del manuscrito. Todos los autores revisaron el manuscrito, acuerdan ser plenamente responsables de garantizar la integridad y precisión del trabajo, y leyeron y aprobaron el manuscrito final.

\section{Conflicto de intereses}

Los autores declaran no tener conflictos de intereses.

\section{Fuente de financiación}

El presente estudio no tuvo financiación.

\section{Referencias bibliográficas}

1. Defrees DN, BaileyJ. Irritable Bowel Syndrome: Epidemiology, Pathophysiology, Diagnosis, and Treatment. Prim Care. 2017;44(4):655-71. doi: 10.1016/j.pop.2017.07.009

2. Oka P, Parr H, Barberio B, Black CJ, Savarino EV, Ford AC. Global prevalence of irritable bowel syndrome according to Rome III or IV criteria: a systematic review and meta-analysis. Lancet Gastroenterol Hepatol. 2020;5(10):908-17. doi: 10.1016/S2468-1253(20)30217-X

3. Chey WD, Kurlander J, Eswaran S. Irritable bowel syndrome: A clinical review. JAMA. 2015;313(9):949-58. doi: 10.1001/ jama.2015.0954

4. Mearin F, Lacy BE, Chang L, Chey WD, Lembo AJ, Simren $\mathrm{M}$, et al. Bowel disorders. Gastroenterology. 2016;S00165085(6)00222-5. doi: 10.1053/j.gastro.2016.02.031

5. López-Colombo A, Morgan D, Bravo-González D, MontielJarquín A, Méndez-Martínez S, Schmulson M. The epidemiology of functional gastrointestinal disorders in Mexico: a population-based study. Gastroenterol Res Pract. 2012;2012:606174. doi: 10.1155/2012/606174

6. Diagnóstico y tratamiento del intestino irritable en el adulto [Internet]. México: CENETEC; 2015 [consultado el 20 de abril de 2021]. Disponible en: https://www.imss.gob.mx/ sites/all/statics/guiasclinicas/042GRR.pdf

7. Enck P, Aziz Q, Barbara G, Farmer AD, Fukudo S, Mayer EA, et al. Irritable bowel syndrome. Nat Rev Dis Primers. 2016;2:16014. doi: 10.1038/nrdp.2016.14

8. Hellström PM. Pathophysiology of the irritable bowel syndrome - Reflections of today. Best Pract Res Clin Gastroenterol. 2019;40-41:101620. doi: 10.1016/j.bpg.2019.05.007

9. Drossman DA, Camilleri M, Mayer EA, Whitehead WE. AGA technical review on irritable bowel syndrome. Gastroenterology. 2002;123(6):2108-31. doi: 10.1053/ gast.2002.37095

10. Talley NJ, Spiller R. Irritable bowel syndrome: a little understood organic bowel disease? Lancet. 2002;360(9332):555-64. doi: 10.1016/S0140-6736(02)09712-X

11. Matricon J, Meleine M, Gelot A, Piche T, Dapoigny M, Muller E, et al. Review article: Associations between immune activation, intestinal permeability and the irritable bowel syndrome. Aliment Pharmacol Ther. 2012;36(11-12):1009-31. doi: 10.1111/apt.12080

12. Saha L. Irritable bowel syndrome: Pathogenesis, diagnosis, treatment, and evidence-based medicine. World J Gastroenterol. 2014;20(22):6759-73. doi:10.3748/wjg.v20. i22.6759

13. Spiller R, Garsed K. Postinfectious irritable bowel syndrome. Gastroenterology. 2009;136(6):1979-88. doi: 10.1053/j.gastro.2009.02.074

14. De Ponti F. Pharmacology of serotonin: what a clinician should know. Gut. 2004;53(10):1520-35. doi:10.1136/ gut.2003.035568

15. Saberi-Firoozi M, Khademolhosseini F, Mehrabani D, Yousefi M, Salehi M, Heidary ST. Subjective lactose intolerance in apparently healthy adults in southern Iran: Is it related to irritable bowel syndrome? Indian J Med Sci. 2007;61(11):591-7. doi: 10.4103/0019-5359.37045

16. Makker J, Chilimuri S, Bella JN. Genetic epidemiology of irritable bowel syndrome. World J Gastroenterol. 2015;21(40):11353-61. doi: 10.3748/wjg.v21.i40.11353

17. Remes-Troche JM, Gómez-Escudero O, Nogueira-de Rojas JR, Carmona-Sánchez R, Pérez-Manauta J, López-Colombo A, et al. Tratamiento farmacológico del síndrome de intestino irritable: revisión técnica. Rev Gastroenterol Mex. 2010;75(1):42-66.

18. Spiller R, Aziz Q, Creed F, Emmanuel A, Houghton L, Hungin $\mathrm{P}$, et al. Guidelines on the irritable bowel syndrome: mechanisms and practical management. Gut. 2007;56(12):1770-98. doi: 10.1136/gut.2007.119446

19. Peralta S, Cottone C, Doveri T, Almasio PL, Craxi A. Small intestinal bacterial overgrowth and irritable bowel syndrome-related symtoms: experience with Rifaximin. World J Gastroenterol. 2009;15(21):2628-31. doi: 10.3748/ wjg. 15.2628 
20. Frissora CL, Cash BD. Review article: the role of antibiotics vs. conventional pharmacotherapy in treating symptoms of irritable bowel syndrome. Aliment Pharmacol Ther. 2007;25(11):1271-81. doi: 10.1111/j.13652036.2007.03313.x

21. Sharara AI, Aoun E, Abdul-Baki H, Mounzer R, Sidani S, Elhajj I. A randomized double-blind placebo-controlled trial of rifaximin in patients with abdominal bloating and flatulence. Am J Gastroenterol. 2006;101(2):326-33. doi: $10.1111 / j .1572-0241.2006 .00458 . x$

22. Sultan S, Malhotra A. Irritable bowel syndrome. Ann Intern Med. 2017;166(11): ITC81-96. doi: 10.7326/ AITC201706060

23. Ramkumar D, Rao SSC. Efficacy and safety of traditional medical therapies for chronic constipation: systematic review. Am J Gastroenterol. 2005;100(4):936-71. doi: 10.1111/j.15720241.2005.40925.x

24. Chapman RW, Stanghellini V, Geraint M, Halphen M. Randomized clinical trial: macrogol/PEG 3350 plus electrolytes for treatment of patients with constipation associated with irritable bowel syndrome. Am J Gastroenterol. 2013;108(9):1508-15. doi: 10.1038/ajg.2013.197

25. Longstreth GF, Thompson WG, Chey WD, Houghton LA, Mearin F, Spiller RC. Functional bowel disorders. Gastroenterology. 2006;130(5):1480-91. doi: 10.1053/j.gastro.2005.11.061

26. McKenzie YA, Bowyer RK, Leach H, Gulia P, Horobin J, O'Sullivan NA, et al. British Dietetic Association systematic review and evidence-based practice guidelines for the dietary management of irritable bowel syndrome in adults (2016 update). J Hum Nutr Diet. 2016;29(5):549-75. doi: 10.1111/ jhn.12385

27. Van Tilburg MAL, Palsson OS, Levy RL, Feld AD, Turner MJ, Drossman DA, et al. Complementary and alternative medicine use and cost in functional bowel disorders: a six month prospective study in a large HMO. BMC Complement Altern Med. 2008;8:46. doi: 10.1186/1472-6882-8-46

28. Camilleri M. Management options for irritable bowel syndrome. Mayo Clin Proc. 2018;93(12):1858-72. doi: 10.1016/j.mayocp.2018.04.032

29. Lea R, Whorwell PJ. The role of food intolerance in irritable bowel syndrome. Gastroenterol Clin North Am. 2005;34(2):247-55. doi: 10.1016/j.gtc.2005.02.005

30. Daley AJ, Grimmett C, Roberts L, Wilson S, Fatek M, Roalfe A, et al. The effects of exercise upon symptoms and quality of life in patients diagnosed with irritable bowel syndrome: a randomised controlled trial. Int J Sports Med. 2008;29(9):77882. doi: $10.1055 /$ s-2008-1038600

31. Van Lanen A-S, de Bree A, Greyling A. Efficacy of a lowFODMAP diet in adult irritable bowel syndrome: a systematic review and meta-analysis. Eur J Nutr. 2021;60(6):3505-22. doi.org/10.1007/s00394-020-02473-0

32. Whelan K, Martin LD, Staudacher HM, Lomer MCE. The low FODMAP diet in the management of irritable bowel syn- drome: an evidence-based review of FODMAP restriction, reintroduction and personalisation in clinical practice. J Hum Nutr Diet. 2018;31(2):239-55. doi: 10.1111/jhn.12530

33. Gibson PR, Shepherd SJ. Evidence-based dietary management of functional gastrointestinal symptoms: The FODMAP approach. J Gastroenterol Hepatol. 2010;25(2):252-8. doi: 10.1111/j.1440-1746.2009.06149.x

34. Whigham L, Joyce T, Harper G, Irving PM, Staudacher HM, Whelan K, et al. Clinical effectiveness and economic costs of group versus one-to-one education for short-chain fermentable carbohydrate restriction (low FODMAP diet) in the management of irritable bowel syndrome. J Hum Nutr Diet. 2015;28(6):687-96. doi: 10.1111/jhn.12318

35. Staudacher HM, Lomer MCE, Anderson JL, Barrett JS, Muir JG, Irving PM, et al. Fermentable carbohydrate restriction reduces luminal bifidobacteria and gastrointestinal symptoms in patients with irritable bowel syndrome. J Nutr. 2012;142(8):1510-8. doi: 10.3945/jn.112.159285

36. Halmos EP, Christophersen CT, Bird AR, Shepherd SJ, Gibson PR, Muir JG. Diets that differ in their FODMAP content alter the colonic luminal microenvironment. Gut. 2015;64(1):93100. doi: $10.1136 /$ gutjnl-2014-307264

37. Tuck C, Barrett J. Re-challenging FODMAPs: the low FODMAP diet phase two. J Gastroenterol Hepatol. 2017;32(Suppl 1):11-5. doi: 10.1111/jgh.13687

38. Probert HM, Gibson GR. Investigating the prebiotic and gas-generating effects of selected carbohydrates on the human colonic microflora. Lett Appl Microbiol. 2002;35(6):473-80. doi: 10.1046/j.1472-765x.2002.01223.x

39. Heizer WD, Southern S, McGovern S. The role of diet in symptoms of irritable bowel syndrome in adults: a narrative review. J Am Diet Assoc. 2009;109(7):1204-14. doi: 10.1016/j.jada.2009.04.012

40. Ford AC, Talley NJ, Spiegel BM, Foxx-Orenstein AE, Schiller L, Quigley EM, et al. Effect of fibre, antispasmodics, and peppermint oil in the treatment of irritable bowel syndrome: systematic review and meta-analysis. BMJ. 2008;337:a2313. doi: 10.1136/bmj.a2313

41. Manning LP, Yao CK, Biesiekierski JR. Therapy of IBS: Is a low FODMAP diet the answer? Front Psychiatry. 2020;11:865. doi: 10.3389/fpsyt.2020.00865

42. Paduano D, Cingolani A, Tanda E, Usai P. Effect of three diets (low-FODMAP, gluten-free and balanced) on Irritable Bowel Syndrome symptoms and health-related quality of life. Nutrients. 2019;11(7):1566. doi: 10.3390/nu11071566

43. Dieterich W, Zopf Y. Gluten and FODMAPS-Sense of a Restriction/When Is Restriction Necessary? Nutrients. 2019;11(8):1957. doi: 10.3390/nu11081957

44. Ford AC, Quigley EMM, Lacy BE, Lembo AJ, Saito YA, Schiller LR, et al. Efficacy of prebiotics, probiotics, and synbiotics in irritable bowel syndrome and chronic idiopathic constipation: systematic review and meta-analysis. Am J Gastroenterol. 2014;109(10):1547-61. doi: 10.1038/ ajg.2014.202 
45. Valdovinos M, Montijo E, Abreu A, Heller S, González-Garay A, Bacarreza D, et al. Consenso mexicano sobre probióticos en gastroenterología. Rev Gastroenterol Mex. 2017;82(2):156178. doi: 10.1016/j.rgmx.2016.08.004

46. Urquiaga I, Echeverría G, Dussaillant C, Rigotti A. Origin, components and mechanisms of action of the Mediterranean diet. Rev Med Chil. 2017;145(1):85-95. doi: 10.4067/S003498872017000100012

47. Tabla orientativa. Alimentos con y sin gluten. [Internet]. Associació Celíacs de Catalunya [consultado el 20 de julio de 2021]. Disponible en: https://www.celiacscatalunya.org/ pdfs/apto_no_apto.pdf 